\title{
Identification of a novel biomarker in tangeretin-induced cell death in AGS human gastric cancer cells
}

\author{
SILVIA YUMNAM ${ }^{1,2^{*}}$, SUCHISMITA RAHA $^{1 *}$, SEONG MIN KIM $^{1}$, \\ VENU VENKATARAME GOWDA SARALAMMA ${ }^{1}$, HO JEONG LEE ${ }^{1}$, SANG EUN HA ${ }^{1}$, \\ JEONG DOO $\mathrm{HEO}^{3}$, SANG JOON LEE ${ }^{3}$, EUN HEE KIM ${ }^{4}$, WON SUP LEE ${ }^{5}$, \\ JIN A. $\mathrm{KIM}^{6}$ and GON SUP KIM ${ }^{1}$
}

\begin{abstract}
${ }^{1}$ Research Institute of Life Science, College of Veterinary Medicine, Gyeongsang National University, Jinju, Gyeongsang 52828; ${ }^{2}$ College of Pharmacy, Gachon University, Incheon 406-799;

${ }^{3}$ Gyeongnam Department of Environment Toxicology and Chemistry, Toxicology Screening Research Center, Korea Institute of Toxicology, Jinju, Gyeongsang 52828; ${ }^{4}$ Department of Nursing Science, International University of Korea, Jinju, Gyeongsang 52833; ${ }^{5}$ Department of Internal Medicine, Institute of Health Sciences, Gyeongsang National University, School of Medicine, Jinju, Gyeongsang 52727; ${ }^{6}$ Department of Physical Therapy, International University of Korea, Jinju, Gyeongsang 52833, Republic of Korea
\end{abstract}

Received December 15, 2017; Accepted September 18, 2018

DOI: $10.3892 /$ or.2018.6730

\begin{abstract}
Proteomic analysis serves as an important biological tool for identifying biological events. Novel biomarkers of a specific disease such as cancer may be identified using these promising techniques. The aim of the present study was to investigate the effect of tangeretin and to identify potential biomarkers in AGS gastric cancer cells using a proteomics approach. The results of the present study revealed that tangeretin inhibited AGS cell viability dose-dependently with a half-maximal inhibitory concentration of $100 \mu \mathrm{M}$. Two-dimensional gel electrophoresis was performed to determine the potential biomarker between control and tangeretin $(100 \mu \mathrm{M})$-treated AGS cells. A total of 16 proteins was identified from 36 significant protein spots using matrix-assisted laser-desorption/ionization time-of-flight-mass spectrometry using peptide fingerprinting. The bioinformatics tools Protein ANalysis THrough Evolutionary Relationships (PANTHER) and Database for Annotation, Visualization and Integrated Discovery (DAVID) were used to identify the functional properties and association of the proteins obtained. Using western blot analysis, the regulatory pattern of four selected proteins,
\end{abstract}

Correspondence to: Professor Gon Sup Kim, Institute of Life Science, College of Veterinary Medicine, Gyeongsang National University, 900 Gazwa, Jinju, Gyeongsang 52828, Republic of Korea E-mail: gonskim@gnu.ac.kr

*Contributed equally

Key words: tangeretin, AGS cells, proteome, two-dimensional gel electrophoresis, protein analysis through evolutionary relationships, database for annotation, visualization and integrated discovery protein kinase $\mathrm{C} \varepsilon$, mitogen-activated protein kinase 4 , phosphoinositide 4-kinase and poly(ADP-ribose) polymerase 14, were successfully verified in replicate sample sets. These selected proteins are primarily involved in apoptosis signaling, angiogenesis, cell cycle regulation, receptor kinase binding, intracellular cytoplasmic and nuclear alterations. Therefore, aim of the present study was to identify potential diagnostic biomarkers from the functional categories of altered protein expression in tangeretin-inhibited AGS gastric cancer cell viability.

\section{Introduction}

Gastric cancer is one of the leading causes of cancer-associated mortality and the fourth most common cancer worldwide. Patients with gastric cancer have a poor prognosis and the 5 -year survival rate is only $20 \%$ (1). Despite the growth of gastric carcinoma being inhibited by chemotherapy, the side effects and toxicity are so high that it becomes intolerable for the majority of patients (2). Therefore, alternative therapeutic drugs are being sought for the treatment of gastric cancer.

Flavonoids are family of polyphenolic compounds that occur naturally in plants. Flavonoids are noted for their biological activities, including antioxidative, anti-inflammatory, anti-allergic and anti-carcinogenic properties. Tangeretin $[5,6,7,8$ tetramethoxy-2-(4-methoxyphenyl)-4H-1-benzopyran-4-one] is a natural $\mathrm{O}$-polymethoxylated flavonoid commonly occurring in fruits of the Citrus genus. Polymethoxylated flavonoids are known to inhibit tumor cell viability more effectively compared with free hydroxylated flavonoids $(3,4)$. It has been identified that tangeretin possesses a number of biological activities such as anti-proliferative, anti-invasive, anti-metastatic and antioxidative properties (5). Tangeretin has been identified to inhibit the viability of breast cancer and colon cancer, and human leukemic cell lines $(6,7)$. Previous study 
has demonstrated that tangeretin induces apoptosis in AGS gastric cancer cells (8). However, to the best of our knowledge, cellular protein alterations in response to tangeretin in AGS gastric cancer cells have not yet been investigated.

Proteomic techniques are promising tools for identifying differentially expressed proteins and they are also able to screen for novel target proteins. Differential proteomics is an important area of proteomics that involves the comparison and identification of proteins that are expressed by a whole genome or in a complex mixture (9). Previous studies have identified that a quantitative proteomic profile reveals markedly abundant differentially expressed proteins that may serve as novel biomarkers on cancer cells that may be targeted using phytonutrients $(10,11)$. The aim of the present study was to identify novel biomarkers for gastric cancer. Despite it having been revealed that tangeretin induces apoptosis in AGS gastric cancer cells (8), to the best of our knowledge, the proteomic profile of tangeretin-induced cell death in AGS cells has not yet been reported.

The aim of the present study was to identify the differentially expressed proteins between tangeretin-treated or untreated AGS cancer cells using a proteomics method. Key functional proteins involved in the major signaling network were identified that revealed the various cellular proteins associated with the regulatory mechanism of cell viability and cell death, which may serve as predictable biomarkers for therapeutic targets.

\section{Materials and methods}

Chemicals and reagents. RPMI-1640 medium, fetal bovine serum (FBS) and antibiotics (streptomycin/penicillin) were purchased from Gibco; Thermo Fisher Scientific, Inc. (Waltham, MA, USA). Materials and chemicals used for electrophoresis were obtained from Bio-Rad Laboratories, Inc. (Hercules, CA, USA). Anti-phosphoinositide 4-kinase (PI4K; $230 \mathrm{kDa}$; cat. no. 4902) and $\beta$-actin (45 kDa; cat. no. 4970) were purchased from Cell Signaling Technology, Inc. (Danvers, MA, USA), anti-mitogen-activated protein kinase 4 (MAPK4; 65 kDa; PA5-14185) was purchased from Thermo Fisher Scientific, Inc., anti-protein kinase C $\varepsilon$ (PKC $\varepsilon$; 90 and 85 kDa; cat. no. 06991) was purchased from Merck \& Co., Inc. (Whitehouse Station, NJ, USA) and anti-poly(ADP-ribose) polymerase 14 (PARP14; $171 \mathrm{kDa}$; cat. no. HPA012063) was purchased from Sigma-Aldrich; Merck KGaA (Darmstadt, Germany). All other chemicals were purchased from Amresco, LLC (Solon, OH, USA) and Sigma-Aldrich; Merck KGaA. The chemicals used were commercially available and of the highest grade.

Cell culture and treatment. The human AGS gastric cancer cell line was obtained from the Korean Cell Line Bank (Seoul, Korea). The cells were maintained in RPMI-1640 medium supplemented with $10 \%$ heat inactivated FBS and $1 \%$ penicillin/streptomycin at $37^{\circ} \mathrm{C}$ in a $5 \% \mathrm{CO}_{2}$ incubator. Cells were treated with vehicle alone [1\% dimethylsulfoxide (DMSO)] or 50, 75, 100 and $150 \mu \mathrm{M}$ tangeretin dissolved in 1\% DMSO.

Cell viability assay. Cell viability was determined using an MTT assay. AGS cells were seeded at a density of
$1 \times 10^{5}$ cells/well in 12 -well plates. Following overnight incubation at $37^{\circ} \mathrm{C}$ in a $5 \% \mathrm{CO}_{2}$ incubator, cells were treated with $0,50,75,100$ and $150 \mu \mathrm{M}$ tangeretin. The MTT assay was performed after $24 \mathrm{~h}$ of incubation. To each well, $100 \mu 10.5 \%$ $(\mathrm{w} / \mathrm{v})$ MTT dissolved in 1X PBS was added prior to incubation at $37^{\circ} \mathrm{C}$ for $3 \mathrm{~h}$. The medium was aspirated and the formazan contained in the cell was solubilized in $500 \mu \mathrm{l}$ DMSO. After $15 \mathrm{~min}$ of shaking, the absorbance at $540 \mathrm{~nm}$ was determined using a microplate reader. Cell viability was expressed as a percentage relative to that of controls (untreated cells), which was set at $100 \%$.

Protein extraction proteins were extracted from AGS cells treated with vehicle or $100 \mu \mathrm{M}$ tangeretin for $24 \mathrm{~h}$. In brief, trypsinized cells were dissolved in lysis buffer containing $7 \mathrm{M}$ urea, $2 \mathrm{M}$ thiourea and 4\% (w/v) 3-[(3-cholamidopropyl) dimethylammonio] propane-1-sulfonic acid (CHAPS). The lysates were centrifuged at $1,000 \times \mathrm{g}$ for $15 \mathrm{~min}$ at $4^{\circ} \mathrm{C}$, and the collected supernatant was stored at $-70^{\circ} \mathrm{C}$ until analysis. Proteins were precipitated with an equal volume (1:1) of $20 \%$ (v/v) trichloroacetic acid and dissolved in $7 \mathrm{M}$ urea, $2 \mathrm{M}$ thiourea and 4\% (w/v) CHAPS, $0.5 \%$ (v/v) immobilized $\mathrm{pH}$ gradient (IPG) buffer and 1\% dithiothreitol (DTT). Protein concentration was then determined using a Non-Interfering ${ }^{\mathrm{TM}}$ Protein Assay kit (G-Biosciences, St. Louis, MO, USA), according to the manufacturer's protocol.

Two-dimensional gel electrophoresis (2DE). IPG strips $(18 \mathrm{~cm}), \mathrm{pH} 3-10$, were rehydrated in a rehydration buffer containing $7 \mathrm{M}$ urea, $2 \mathrm{M}$ thiourea, 4\% (w/v) CHAPS and $0.002 \%$ bromophenol blue. For the first dimension, $800 \mu \mathrm{g}$ protein was focused using the Ettan IPG Phor II isoelectric focusing (IEF) system (GE Healthcare, Chicago, IL, USA) at $50 \mathrm{~V}$ for $1 \mathrm{~h}$, followed by $200 \mathrm{~V}$ for $1 \mathrm{~h}, 400 \mathrm{~V}$ for $30 \mathrm{~min}$, $500 \mathrm{~V}$ for $30 \mathrm{~min}, 4,000 \mathrm{~V}$ for $1 \mathrm{~h}$ for hold, 4,000 V for $1 \mathrm{~h}$ for gradient, $10,000 \mathrm{~V}$ for $1 \mathrm{~h}, 10,000 \mathrm{~V}$ for $13 \mathrm{~h}$ and $50 \mathrm{~V}$ for $3 \mathrm{~h}$. The focused strips were equilibrated twice for $15 \mathrm{~min}$ each, first with $10 \mathrm{mg} / \mathrm{ml}$ DTT and then with $40 \mathrm{mg} / \mathrm{ml}$ iodoacetamide (IAA) prepared in equilibration buffer containing $50 \mathrm{mM}$ Tris/ $\mathrm{HCl}$ (pH 8.8), $6 \mathrm{M}$ urea, 30\% (v/v) glycerol, 2\% $(\mathrm{w} / \mathrm{v})$ SDS and $0.002 \%(\mathrm{w} / \mathrm{v})$ bromophenol blue. The focused proteins were then separated in the second dimension by SDS-PAGE (12\% linear gradient) with a constant current of $15 \mathrm{~mA} / \mathrm{gel}$ at $20^{\circ} \mathrm{C}$ until the dye reached the bottom of the gel.

Gel spot detection and in-gel digestion. Silver staining was performed for protein spot visualization. Three independent gels were stained in triplicate. Scanned gel images were acquired using a GS-800 scanner (Bio-Rad Laboratories, Inc.) and imported into Progenesis SameSpots software (version 4.1; Nonlinear Dynamics, Ltd., Newcastle upon Tyne, UK) for differential spot expression analysis using automatic matching alignment of the detected protein spots. Spots differing significantly $(\mathrm{P}<0.05$ and $\mathrm{P}<0.1)$ in their intensities with a fold-change $\geq 1.5$ were used for further analysis. Selected protein spots were excised manually from the two-dimensional electrophoresis (2DE) gel and protein digestion was performed according to a silver stain gel extraction protocol (12) with a slight modification. Briefly, the excised gel pieces were washed with deionized water prior 
to destaining with $30 \mathrm{mM}$ potassium ferricyanide for $10 \mathrm{~min}$ until the silver stain disappeared followed by washing three times with deionized water for 5 min each and dehydration in $100 \mu \mathrm{l}$ acetonitrile for $10 \mathrm{~min}$. Dehydrated pieces were dried in a lyophilizer (SFDSM06; Samwon Freezing Engineering Co., Busan, Korea), the gel pieces were rehydrated in $100 \mu \mathrm{l}$ $100 \mathrm{mM} \mathrm{NH}_{4} \mathrm{HCO}_{3}$ continuing the reduction (10 mM DTT) and alkylation (100 mM IAA) process at room temperature for 45 min. Following simultaneous drying and rehydrating, and vacuum drying, the gel pieces were trypsinized with $20 \mathrm{ng} / \mu \mathrm{l}$ trypsin (Promega Corporation, Madison, WI, USA) on ice. After $45 \mathrm{~min}, 10-20 \mu 150 \mathrm{mM} \mathrm{NH} \mathrm{HCO}_{3}$ was added followed by overnight digestion at $37^{\circ} \mathrm{C}$. These peptide mixtures were extracted for subsequent steps of matrix-assisted laser-desorption ionization (MALDI) spot targeting.

MALDI-time-of-flight (TOF)-mass spectrometry (MS) and tandem MS (MS/MS) analysis. The aforementioned pooled extracts were dried in a lyophilizer and the extracts were redissolved in $1 \mu \mathrm{l}$ extraction buffer (50 $\mu \mathrm{l}$ acetonitrile, $20 \mu \mathrm{l}$ trifluoroacetic acid and $930 \mu \mathrm{l}$ distilled water) and $1 \mu \mathrm{l}$ matrix solution ( $\alpha$-acyano-4-hydroxycinnamic acid) and targeted onto a MALDI-TOF plate. Following drying the samples completely onto the targeting plate, MALDI-TOF-MS was performed using a Voyager-DE STR mass spectrometer (Applied Biosystems; Thermo Fisher Scientific, Inc.) equipped with delay ion extraction. Mass spectra were acquired over a mass range between 800 and 3,000 Da. The peptide mass peak list was processed using DataExplorer software (version 4.8; Applied Biosystems; Thermo Fisher Scientific, Inc.) to search the protein against the SwissProt database (www.ebi. ac.uk/uniprot) using the Mascot-Peptide Mass Fingerprint program (www.matrixscience.com). The following parameters were used for database searches: Taxonomy, Homo sapiens (human); cleavage specificity, trypsin with one missed cleavage allowed; peptide tolerance of 100 p.p.m. for the fragment ions; and allowed modifications, cysteine carbamidomethyl (fixed) and oxidation of methionine (variable). The MOWSE scores $(>56)$ and species were considered to identify the correct protein from the Mascot results list.

Bioinformatics analysis. Functional genome ontology of the identified proteins was performed using Protein ANalysis THrough Evolutionary Relationships (PANTHER; version 11.1; pantherdb.org) database. PANTHER uses GO-Slim which is a subset of Gene Ontology (GO). Proteins were further annotated using Database for Annotation, Visualization and Integrated Discovery (DAVID) gene bioinformatics resource for enrichment analysis and association (version 6.8; david.ncifcrf.gov). Expression Analysis Systematic Explorer (EASE) was used for the biological interpretation of the genes derived from the proteomics profile. Protein interactions were identified using Search Tool for the Retrieval of Interacting Genes/Proteins (STRING; version 10) database (13). Markov Cluster Algorithm (MCL) is used for clustering the proteins that were displayed in the network.

Western blot analysis. Briefly, AGS cells treated with vehicle or $100 \mu \mathrm{M}$ tangeretin for $24 \mathrm{~h}$ and were lysed overnight

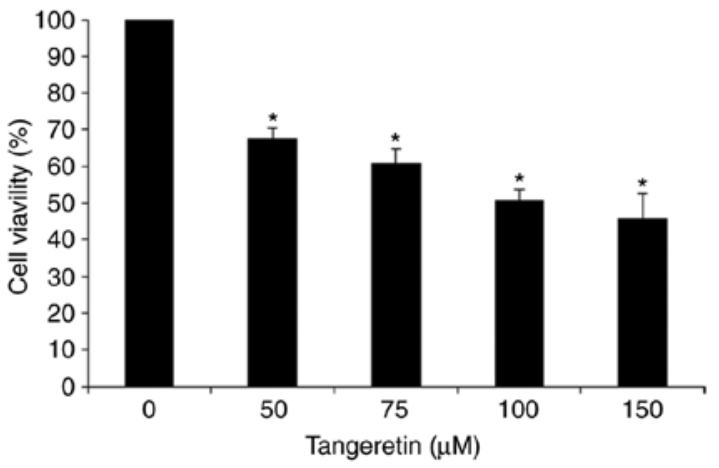

Figure 1. Effect of tangeretin on AGS cell viability. AGS cells were treatedx with the indicated concentration of tangeretin for $24 \mathrm{~h}$ and an MTT assay was performed. ${ }^{*} \mathrm{P}<0.05$ vs. untreated control.

with lysis buffer (radioimmunoprecipitation assay buffer) containing phosphatase inhibitor cocktail along with protease inhibitor and EDTA (Thermo Fisher Scientific, Inc.). The extracted proteins were then centrifuged at $1,000 \mathrm{x} \mathrm{g}$ for $30 \mathrm{~min}$ at $4^{\circ} \mathrm{C}$ to remove debris. Amounts of $20 \mu \mathrm{g}$ proteins, determined using the Bradford assay, were resolved by SDS-PAGE (8-12\% gel) and subsequently transferred onto a polyvinylidene difluoride membrane (Immobilon-P, $0.45 \mu \mathrm{m}$; EMD Millipore, Billerica, MA, USA) using a TE 77 Semi-Dry Transfer Unit (GE Healthcare Life Sciences, Little Chalfont, UK). The membranes were blocked with $5 \%$ bovine serum albumin (BioShop Canada Inc., Burlington, Canada) in Tris-buffered saline containing 1\% Tween-20 (TBS-T, pH 7.4) or 1X Phospho blocking solution (TransLab Biosciences, Daejon, Korea) at room temperature for $1 \mathrm{~h}$. Blots were probed with 1:500 (anti-PI4K and anti-PKCع) or 1:1,000 (anti-MAPK4 and anti-PARP14) dilutions of the respective primary antibodies at $4^{\circ} \mathrm{C}$ for overnight. Following washing five times with TBS-T, the membranes were incubated with horseradish peroxidase-linked anti-rabbit IgG (cat. no. 7074; Cell Signaling Technology, Inc.) secondary antibodies diluted 1:1,000 (for detection of PI4K and PKCE) or 1:2,000 (for detection of MAPK4, PARP14 and $\beta$-actin) at room temperature for $3 \mathrm{~h}$. The immunoblots were visualized using an enhanced chemiluminescence kit and western blotting detection reagents (GE Healthcare Life Sciences). Each protein band was quantified densitometrically using ImageJ software (version 1; imagej. nih.gov/ij; National Institutes of Health, Bethesda, MD, USA) following normalization to $\beta$-actin expression.

Statistical analysis. Results are expressed as the mean \pm standard deviation of a minimum three replicates in independent experiments. The data were analyzed using one-way analysis of variance followed by a Newman-Keuls post hoc test using GraphPad Prism (version 5; GraphPad Software, Inc., La Jolla, CA, USA). $\mathrm{P}<0.05$ was considered to indicate a statistically significant difference.

\section{Results}

Effect of tangeretin on AGS cell viability. In order to assess the effect of tangeretin on the viability of AGS cells, an MTT assay was performed. It was observed that tangeretin treatment 


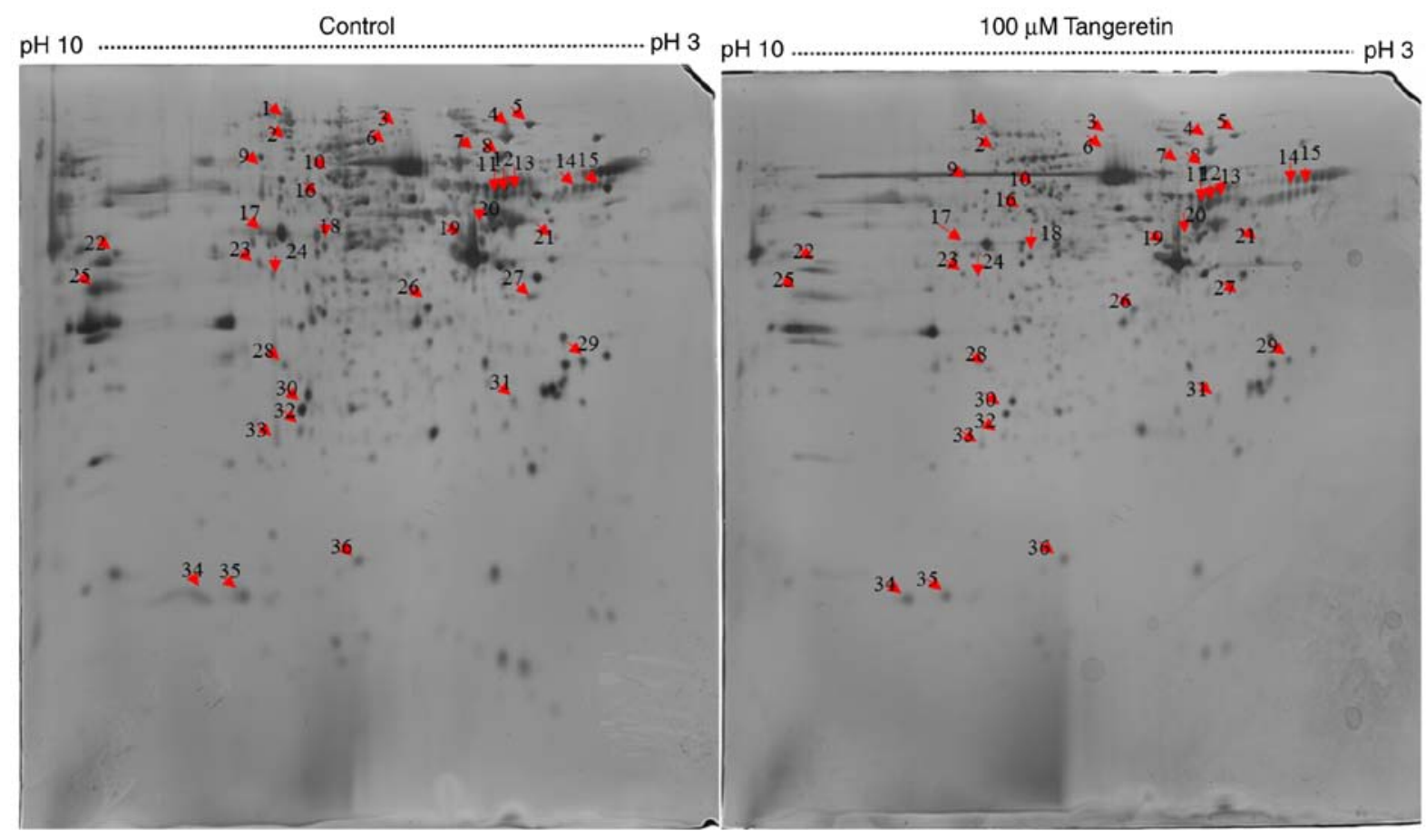

Figure 2. Two-dimensional protein map of AGS cells treated with tangeretin. Proteins were separated on $18 \mathrm{~cm} \mathrm{pH} \mathrm{3-7} \mathrm{immobilized} \mathrm{pH} \mathrm{gradient} \mathrm{strips} \mathrm{for}$ the first dimension and by SDS-PAGE (12\% gel) for the second dimension. The red arrows indicate the 36 differentially expressed protein spots, subsequently identified using matrix-assisted laser-desorption ionization-time-of-flight-mass spectrometry.

decreased the cell viability of AGS cells dose-dependently with an $\mathrm{IC}_{50}$ value of $100 \mu \mathrm{M}$ (Fig. 1). This result suggests that tangeretin induced significant inhibition and cell death in AGS cells, and the $100 \mu \mathrm{M}$ concentration of tangeretin was selected for further experiments.

Proteomic analysis of AGS cells in response to tangeretin treatment. To analyze the underlying molecular mechanism of tangeretin-induced AGS cell death, $800 \mu \mathrm{g}$ total proteins were separated by IEF on $18 \mathrm{~cm}$ IPG strips in the first dimension and resolved by $2 \mathrm{DE}$ followed by silver staining for visualization. A total of 300 protein spots were identified, with 36 spots differing significantly in their intensities with a fold change $\geq 1.5$ (Fig. 2). These 36 differentially expressed proteins spots were selected for further analysis using MALDI-TOF-MS. As MALDI-TOF-MS detects fewer peptides, these 36 differentially expressed spots were analyzed further using the MASCOT search engine. Owing to post-translational modification or proteolytic cleavage, a number of proteins may be detected from one spot or the same protein may be detected from different spots. Of the 38 spots, 16 significantly differentially expressed proteins were successfully identified using the MASCOT search engine, and the SwissProt database revealed two upregulated proteins and 14 downregulated proteins (Table I).

Functional classification of identified proteins. The PANTHER database was used to analyze the 16 identified proteins in terms of molecular function, biological process, cellular component, protein class and pathway (Fig. 3). The most common molecular functions were binding protein $(41.7 \%)$, catalytic activity $(41.7 \%)$, signal transducer activity $(8.3 \%)$ and structural molecular activity $(8.3 \%)$. The major biological processes were cellular process $(29 \%)$, metabolic process $(22.6 \%)$, biological regulation (12.9\%), biogenesis (12.9\%), stimulus (12.9\%), developmental process (3.2\%), multicellular organismal process $(3.2 \%)$ and reproduction (3.2\%). The cell component carries cell part (46.7\%), organelle (26.7\%), macromolecular complex (20.0\%) and cell junction (6.7\%). Regarding the protein class, the most common were transferase (26.7\%), transfer/carrier protein $(20.0 \%)$, calcium-binding protein $(13.3 \%)$, hydrolase $(13.3 \%)$, chaperone $(6.7 \%)$, enzyme modulator $(6.7 \%)$, cytoskeleton protein $(6.7 \%)$ and receptor $(6.7 \%)$.

PANTHER classification identified 21 pathways with signaling mechanisms that are involved in the effect of tangeretin on AGS cancer cells (Fig. 4). Among the 16 derived proteins, PKCE (encoded by KPCE) is the major protein leading the cellular signaling mechanism in the obtained pathways (Table II).

In order to obtain maximum comparable classification between the obtained proteins, DAVID enrichment analysis was performed, which identified four clustering annotation groups with medium classification stringency (Fig. 5). Markedly associated genes in functional annotations were identified on the basis of threshold count $\geq 2$ and EASE $<0.1$. It has been observed that the majority of genes are associated with cell-cell adhesion in the first cluster with enrichment score 1.96 and in the second cluster of nucleotide binding and kinase activity with enrichment score 1.89 . The majority of genes are associated with cell adhesion and junction, nucleotide and ATP binding, transferase and kinase activity, nucleus and transcription regulation, cell and plasma membrane. 


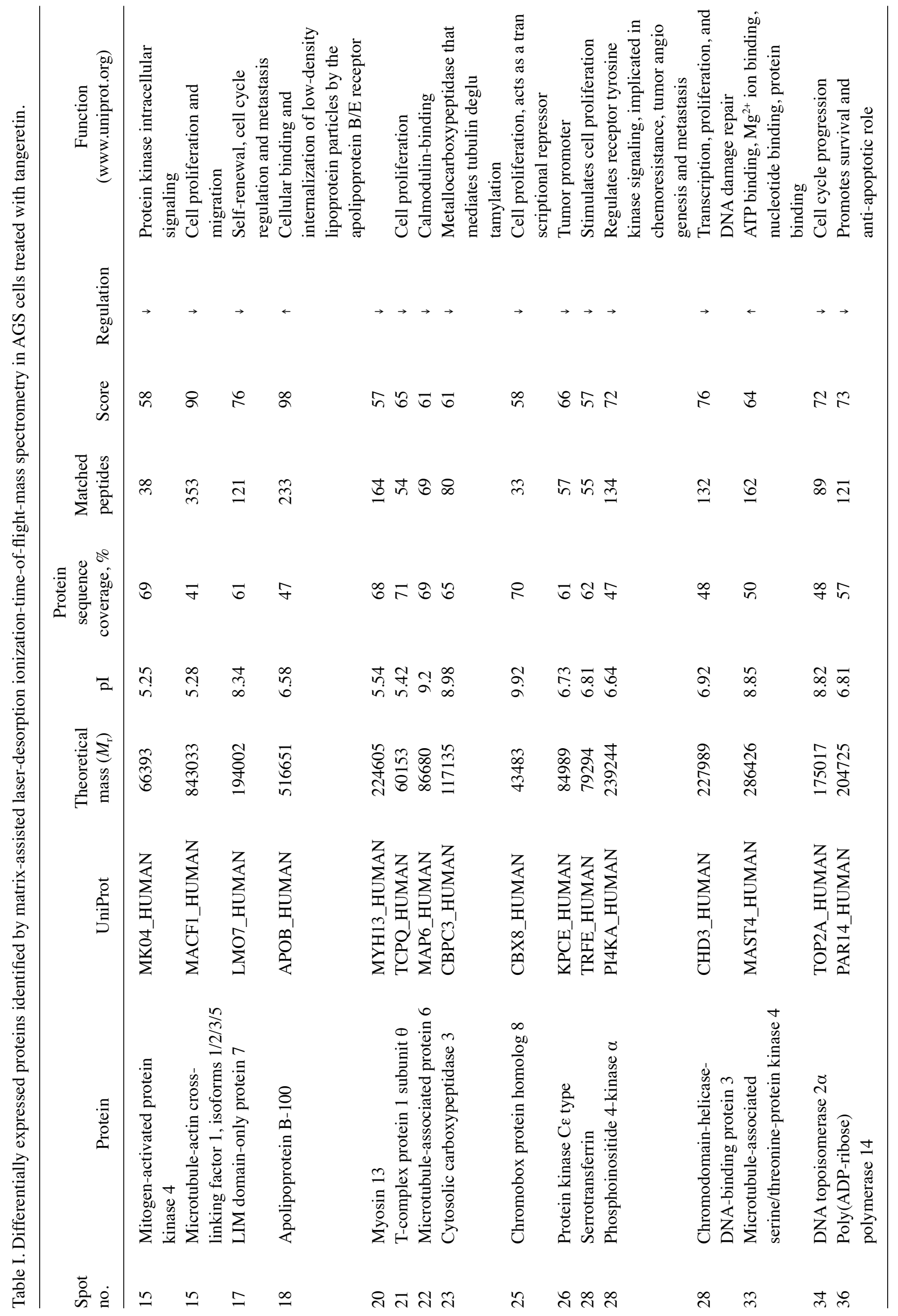




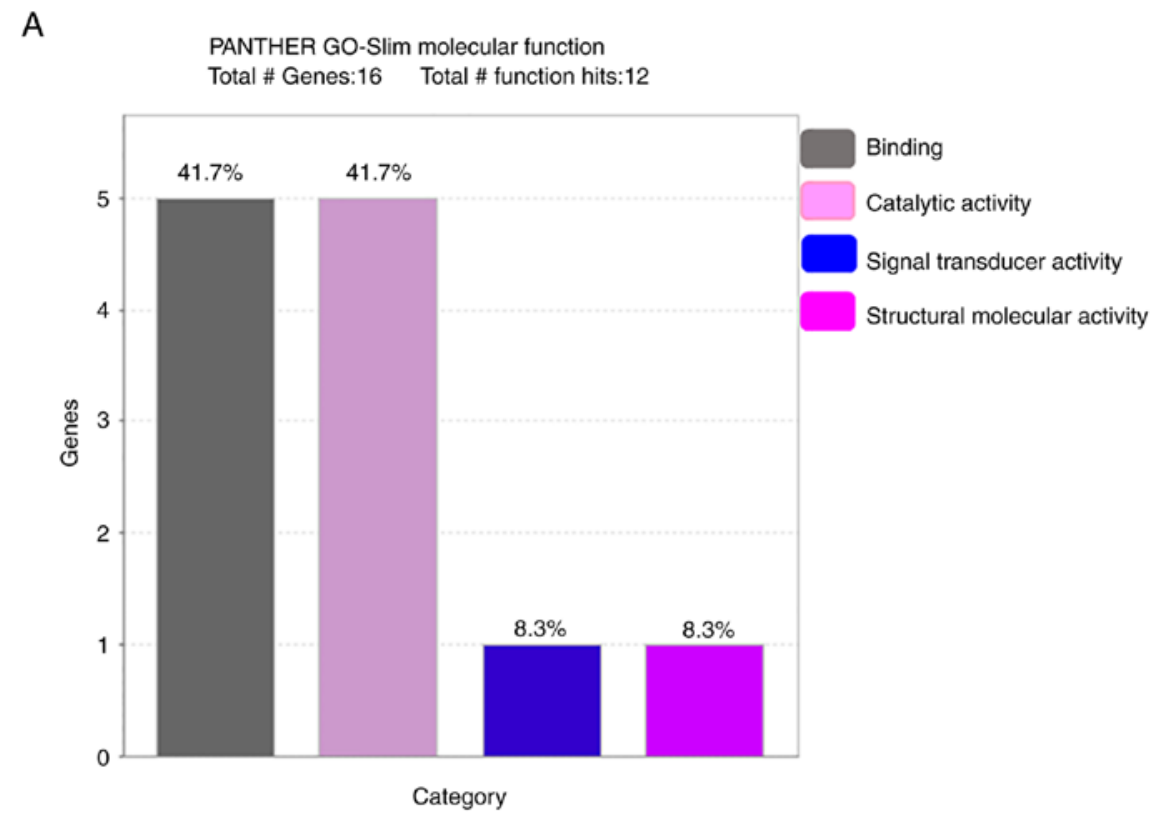

B

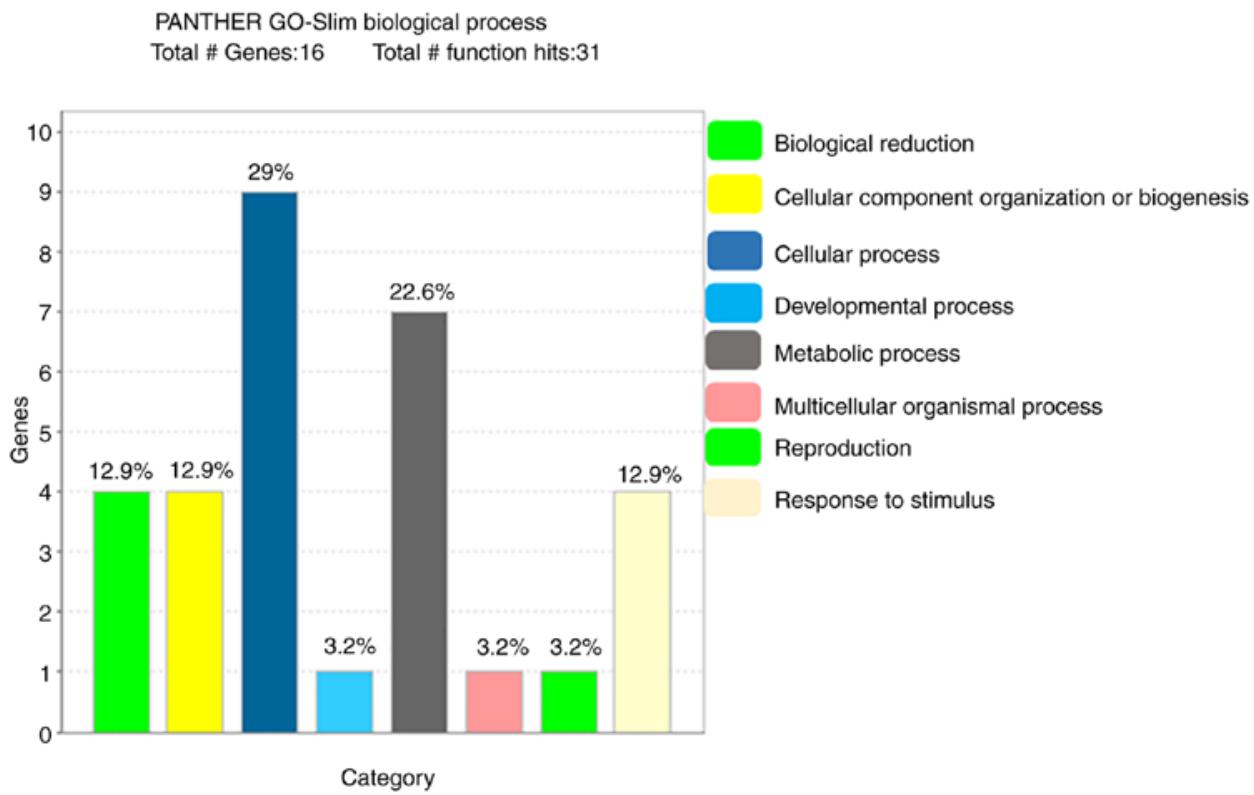

Figure 3. Classification of differentially expressed proteins. A total of 16 differentially expressed proteins were classified according to (A) molecular function, (B) biological process using the PANTHER database. Functional percentage of each category refers to the gene hit against the total number of functional hits.

Interaction between protein complexes. The selected genes were interrogated using the STRING database for the protein-protein interaction network analysis between the upregulated and downregulated proteins. STRING generated an interconnected protein network with a medium confidence level 0.04, developed three signaling modules including two database-predicted nodes or genes following clustering using the MCL. These three modules included PKC $\varepsilon$ protein regulating MAPK4 and PI4KA; apolipoprotein (APOB) interacting with transferrin transfer protein; and chaperonin containing T-complex 1 (TCP1) (CCT) 8 gene interacting with STRING-predicted TCP1 subunits CCT2, CCT3, CCT4 and CCT5, those again interact directly with DNA topoisomerase II $\alpha$ (TOP2A) and chromodomain helicase DNA-binding protein 3 (CHD3) (Fig. 6).

Validation of selected proteins by western blot analysis. Among the various protein spots identified using MALDI-TOF-MS, four proteins, namely MAPK4, PI4K, PARP14 and PKC , were selected on the basis of MASCOT analysis, PANTHER and DAVID database tool. These protein expressions were confirmed further by western blot analysis. As presented in Fig. 7, the expression of MAPK4, PI4K, PARP14 and PKC $\varepsilon$ in the presence of tangeretin was significantly decreased compared with the untreated condition, thereby confirming the involvement of these proteins from the differentially expressed $2 \mathrm{DE}$ analysis. 


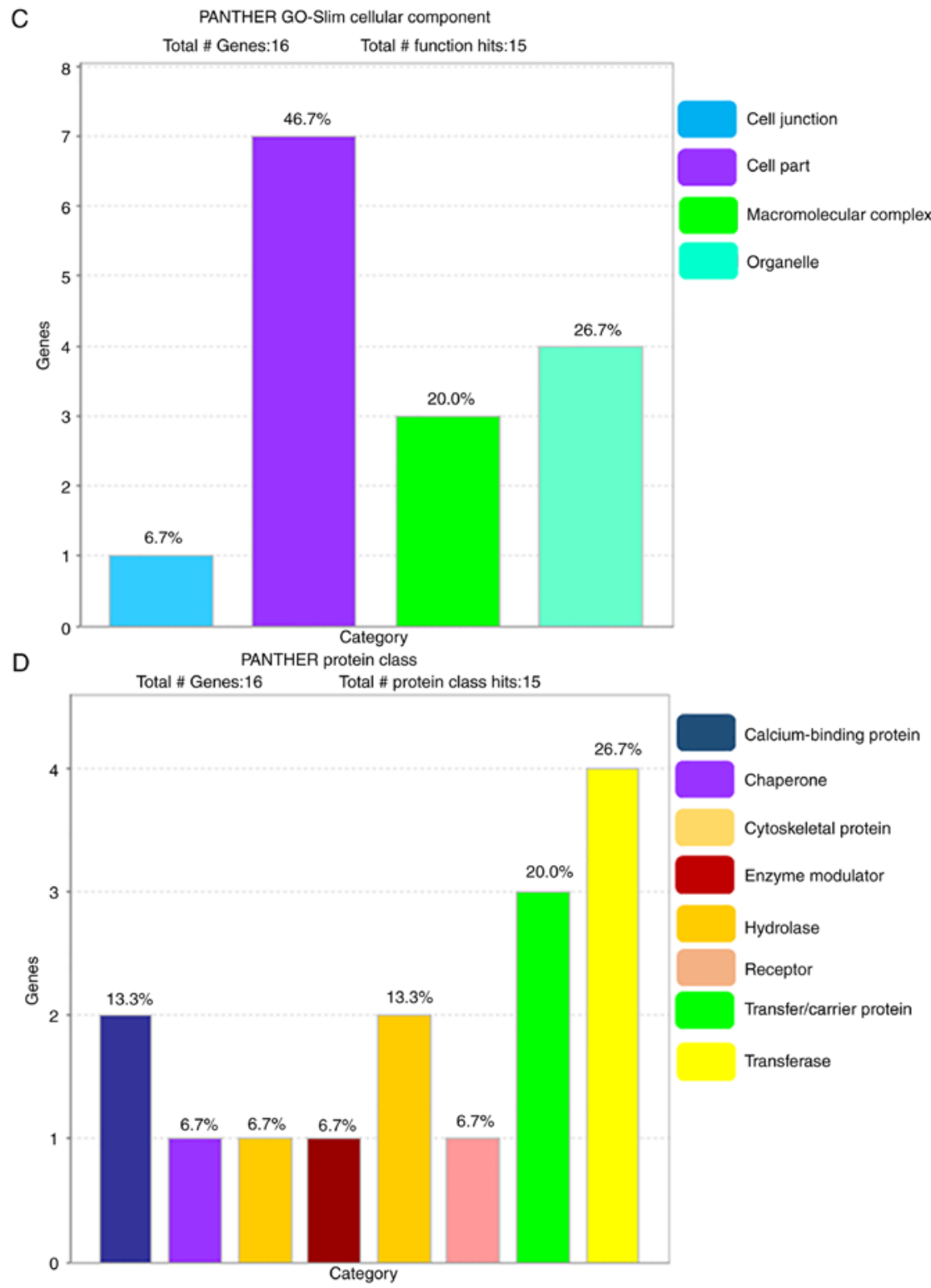

Figure 3. Continued. Classification of differentially expressed proteins. A total of 16 differentially expressed proteins were classified according to (C) cellular component and (D) protein class using the PANTHER database. Functional percentage of each category refers to the gene hit against the total number of functional hits. PANTHER, Protein ANalysis THrough Evolutionary Relationships; GO, Gene Ontology.

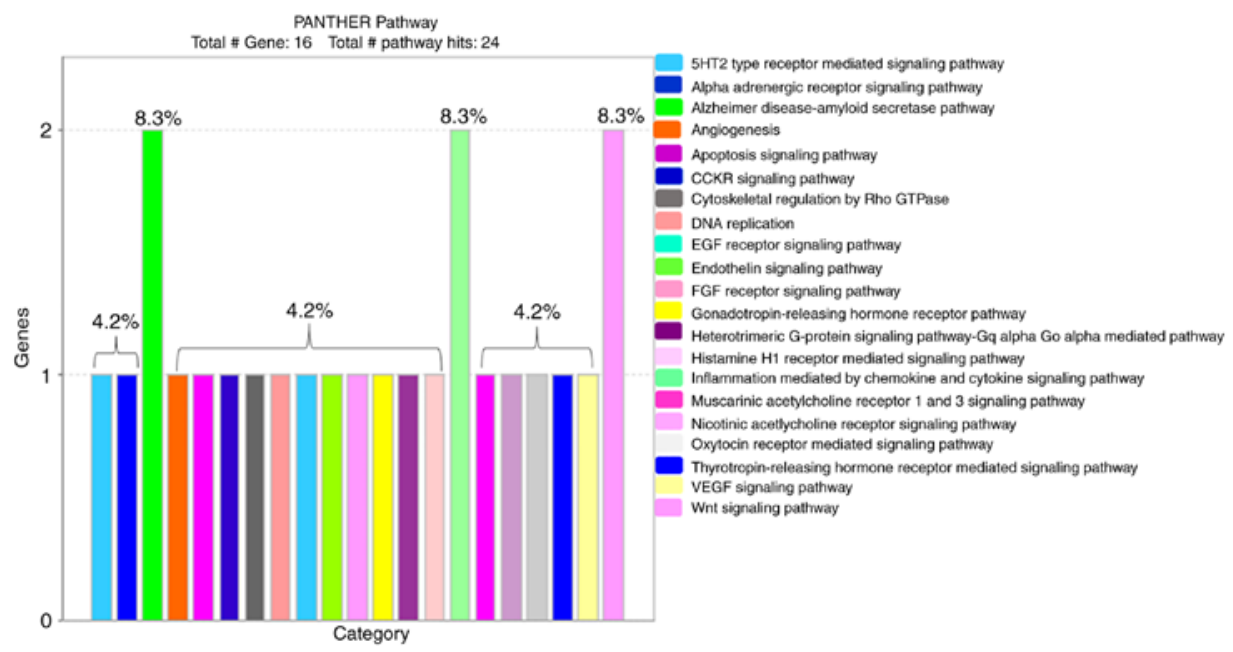

Figure 4. Functional cell signaling pathways. The PANTHER tool identified 21 signaling pathways in tangeretin-treated AGS cells. PANTHER, Protein ANalysis THrough Evolutionary Relationships; 5HT2, 5-hydroxytryptamine 2; CCKR, cholecystokinin; EGF, epidermal growth factor; FGF, fibroblast growth factor; VEGF, vascular endothelial growth factor. 


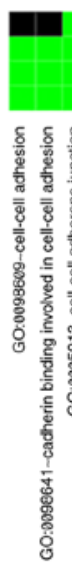

LIM domain 7 (LM07) Phosphatidylinositol 4-kinase alpha (PI4KA) Microtubule-actin crosslinking factor 1 (MACF1)

Chaperonin containing TCP1 subunit 8 (CCT8)

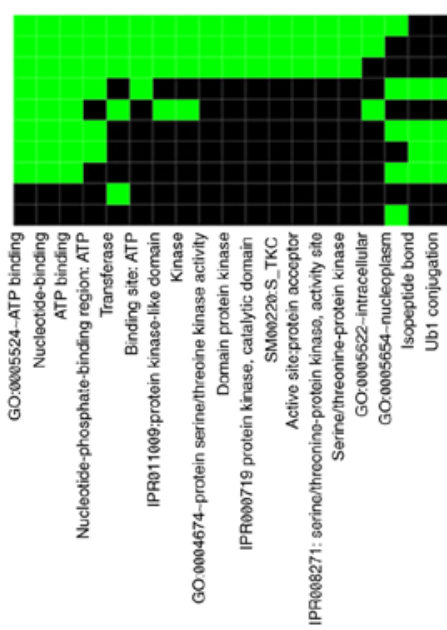

Mitogen-activated protein kinase 4 (MAPK4) Microtubule associated serine/threonine kinase family member 4 (MAST4) Protein kinase C epsilon (PRKCE)

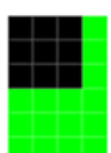

Cluster 1

Cluster 2

Topoisomerase (DNA) II alpha (TOP2A) Mitogen-activated protein kinase 4 (MAPK4) Protein kinase $\mathrm{C}$ epsilon (PRKCE) Poly (ADP.ribose) polymerase tamily member 14 (PARP14) Chromobox 8(CBX8)

Chromodomain hellicase DNA binding protein 3 (CHD3)

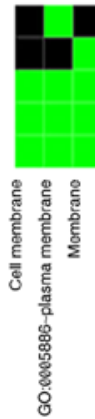

Chromodomain helicase DNA binding protein 3 (CHD3)

Myosin heavy chain 13 (MYH13)

Cheronin containing TCP1 subunt 8 (CCT8)

Poly (ADP.ribose) polymerase family member 14 (PARP14)

Cluster 3

Cluster 4

Figure 5. Clusters identified using DAVID functional annotation. Four clusters were identified using the DAVID functional annotation clustering tool Annotated cluster represented a $\kappa$ value $>0.35$ and overlap $=3$. Similarity scores ranged from high $(>1)$ to low enrichment $(<0.25)$. DAVID, Database for Annotation, Visualization and Integrated Discovery.

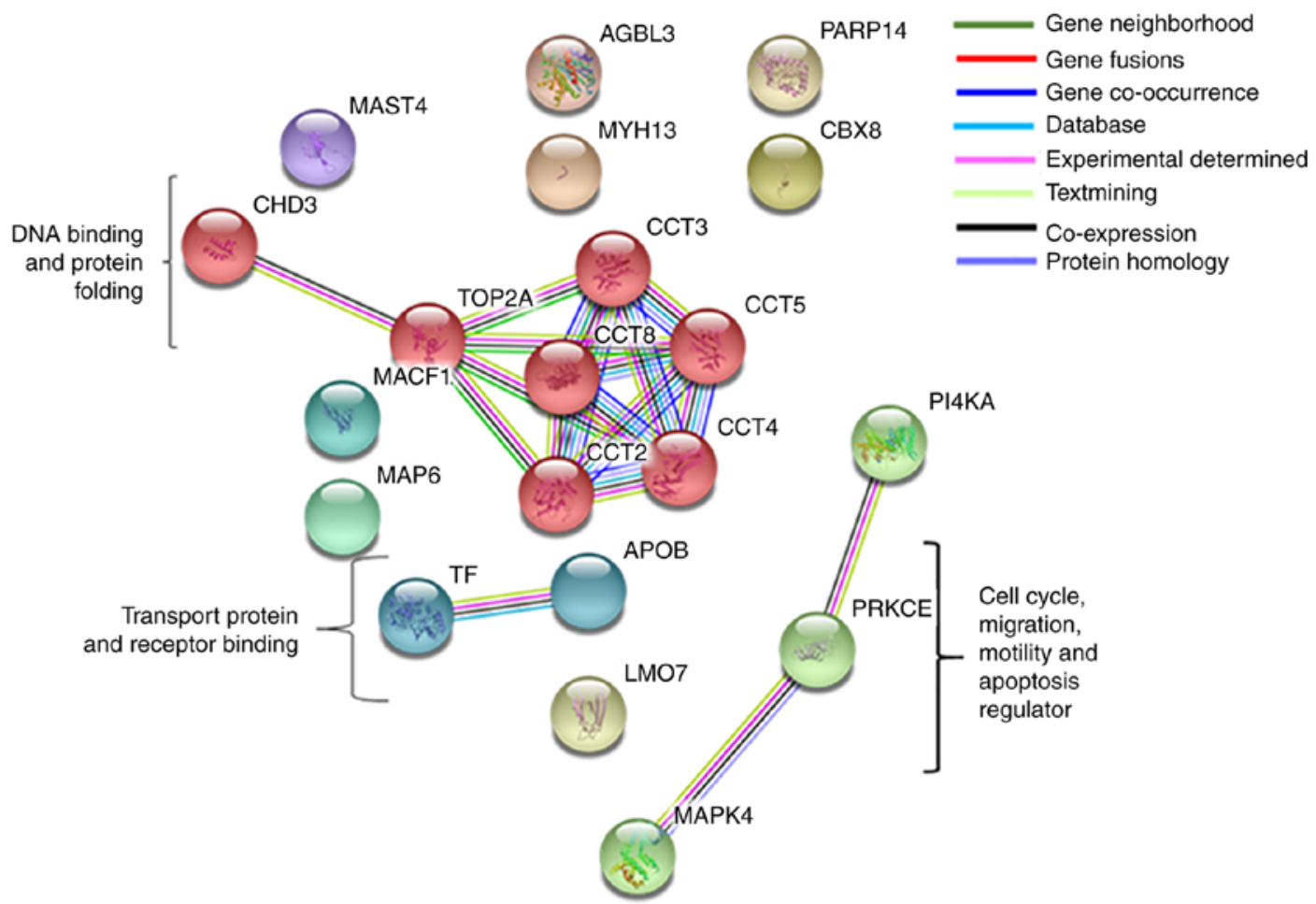

Figure 6. Protein interaction using STRING. Interaction between proteins complex through the STRING database formed three modules using Markov Cluster Algorithm means. The number of lines represents strength of predicted functional interactions between proteins. STRING, Search Tool for the Retrieval of Interacting Genes/Proteins. 
Table II. Signaling pathways and the regulating genes in tangeretin-treated AGS cells using the Protein ANalysis THrough Evolutionary Relationships (PANTHER) database.

\begin{tabular}{|c|c|c|}
\hline No. & Pathway & Symbol of regulated gene \\
\hline 1 & 5-Hydroxytryptamine type 2 receptor-mediated signaling pathway & KPCE \\
\hline 2 & $\alpha$-adrenergic receptor signaling pathway & $\mathrm{KPCE}$ \\
\hline 3 & Alzheimer's disease-amyloid secretase pathway & MK04, KPCE \\
\hline 4 & Angiogenesis & KPCE \\
\hline 5 & Apoptosis signaling pathway & KPCE \\
\hline 6 & Cholecystokinin receptor signaling map & $\mathrm{KPCE}$ \\
\hline 7 & Cytoskeletal regulation by Rho GTPase & MYH13 \\
\hline 8 & DNA replication & TOP2A \\
\hline 9 & Epidermal growth factor receptor signaling pathway & KPCE \\
\hline 10 & Endothelin signaling pathway & KPCE \\
\hline 11 & Fibroblast growth factor signaling pathway & KPCE \\
\hline 12 & Gonadotropin releasing hormone receptor pathway & KPCE \\
\hline 13 & Heterotrimeric G-protein signaling pathway: $\mathrm{G}_{\mathrm{q} \alpha}$ and $\mathrm{G}_{\mathrm{o} \alpha}-$ mediated pathway & KPCE \\
\hline 14 & Histamine $\mathrm{H}_{1}$ receptor-mediated signaling pathway & KPCE \\
\hline 15 & Inflammation mediated by chemokine and cytokine signaling pathway & KPCE, MYH13 \\
\hline 16 & Muscarinic acetylcholine receptor 1 and 3 signaling pathway & KPCE \\
\hline 17 & Nicotinic acetylcholine receptor 1 and 3 signaling pathway & MYH13 \\
\hline 18 & Oxytocin receptor-mediated signaling pathway & $\mathrm{KPCE}$ \\
\hline 19 & Thyrotropin-releasing hormone receptor signaling pathway & KPCE \\
\hline 20 & Vascular endothelial growth factor signaling pathway & $\mathrm{KPCE}$ \\
\hline 21 & Wnt signaling pathway & KPCE, MYH13 \\
\hline
\end{tabular}
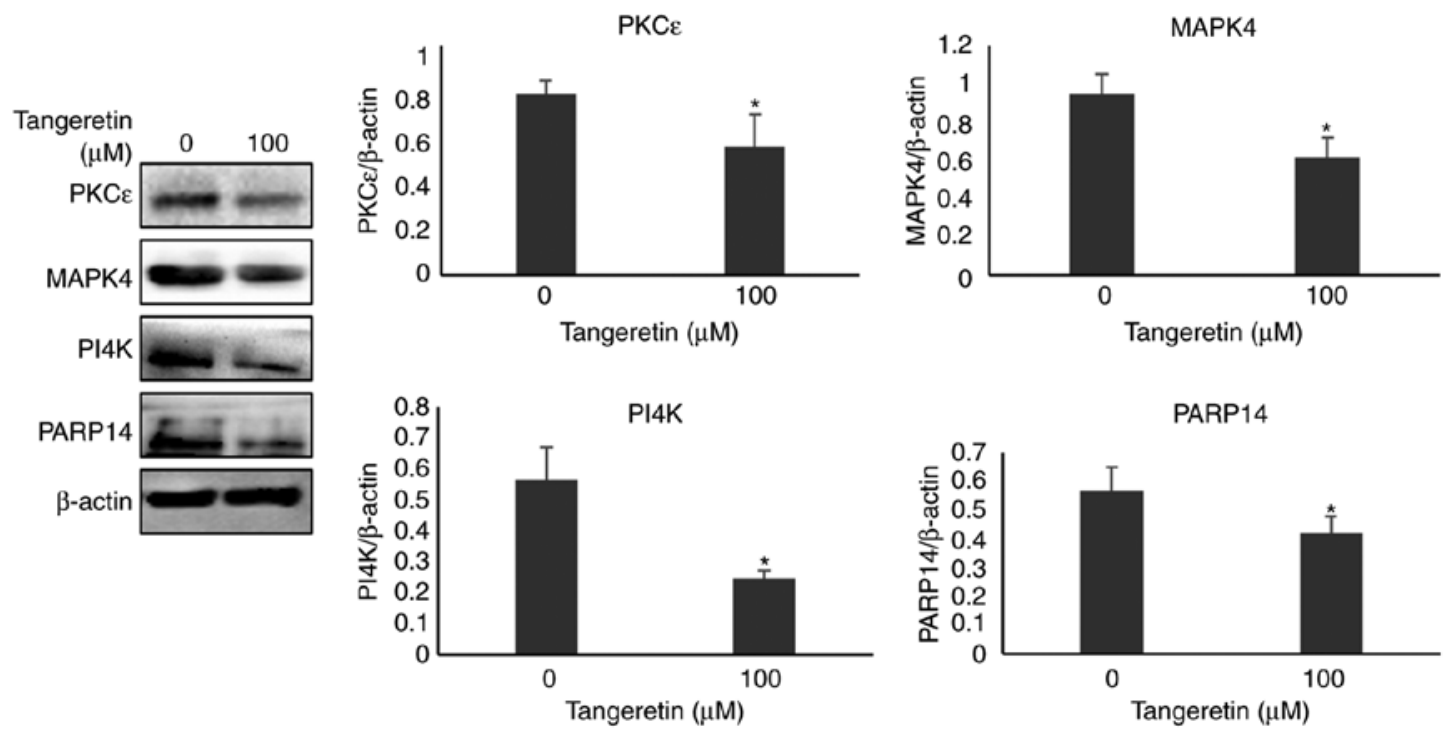

Figure 7. Protein expression of PKC $\varepsilon$, MAPK4, PI4K and PARP14. PKC $\varepsilon$, MAPK4, PI4K and PARP14 protein expression in AGS cells treated with or without tangeretin $(100 \mu \mathrm{M})$ was determined using western blotting. ${ }^{*} \mathrm{P}<0.05$ vs. untreated control. PKC $\varepsilon$, protein kinase C $\varepsilon$; MAPK4, mitogen-activated protein kinase 4; PI4K, phosphoinositide 4-kinase; PARP14, poly(ADP-ribose) polymerase 14.

\section{Discussion}

Tangeretin is a polymethoxylated flavonoid with anti-proliferative, anti-invasive, anti-metastasis and anticancer properties $(4,6,8)$. In the present study, for the first time, proteomic analysis of AGS gastric cancer cells treated with tangeretin was performed. Proteomic analysis data indicated differential expression of 36 spots representing 16 different proteins which were identified using MASCOT search engine analysis. PANTHER and DAVID bioinformatics tools were used to identify the functional properties of these differentially expressed proteins. Exploring the protein-protein interaction 
networks may suggest novel directions for future experimental research and provide cross-species predictions for efficient interaction mapping. The identified proteins represent several biological functions already known in different cancer studies. However, attention towards differential proteome expression analysis on gastric cancer using flavonoids for the identification of functional biomarkers has not been investigated to any great extent.

PANTHER is part of the Gene Ontology Phylogenetic Annotation Project. PANTHER classification categories involved marked proteins enriched with protein binding; microtubule motor, kinase and hydrolase activity. Tight control of cell proliferation and morphogenesis in conjunction with programmed cell death (apoptosis) is required to ensure normal tissue or cell patterning. Imbalance in the cellular signal mechanism, promotes cell proliferation, suppress apoptosis and enhance cell invasion. Receptor protein binding such as with tyrosine kinases serves a detrimental function in cancer cell development, with alterations in the receptor tyrosine kinase potentially leading to the creation of potent oncogenes (14). Intermediate filaments and microtubule cytoskeleton protein binding are the key functions that underpin cellular processes such as disruption of cellular morphogenesis, inappropriate migration and invasion, and genome instability by accompanying the progression of disease (15). Studies have revealed that $\mathrm{G}$-proteins regulate a number of cellular functions including cytoskeletal rearrangement, cell motility, intracellular trafficking, transcriptional regulation, cell viability and development (16). Microtubule motor proteins regulate kinesin superfamily members and support a number of cellular functions, including mitosis, meiosis and the transport of macromolecules (17). Kinases are important mediators of the signaling cascade and oncogenic activation in cancer cells may be blocked by selective kinase inhibitors (18). Calcium-binding protein or calmodulin serves a major function in eukaryote cell signaling, including cell proliferation, programmed cell death and autophagy (19). Similarly, in the present study, receptor binding protein MAPK4, cytoskeleton microtubule actin cross-linking factor 1 (20) and microtubule-associated protein 6 are downregulated. In addition cell cycle regulator TOP2A; intracellular cytoplasmic protein PI4KA, chaperone protein TCP1 subunit $\theta$ (TCPQ), kinase activity protein PKC , PI4KA, cell adhesion junction protein LM07, nuclear protein PARP14, transcriptional repressor chromobox protein homolog 8 (21), DNA and RNA binding CHD3, G protein and metalloprotease cytosolic carboxypeptidase 3 and transferrin protein serrotransferrin (22) were downregulated, whereas microtubule-associated serine/threonine-protein kinase 4 and APOB were upregulated.

PKCE was identified to be major linking protein in tangeretin-induced AGS cell death (23). Overexpression of PKC $\varepsilon$ has been reported in a number of types of cancer. In glioma, skin carcinoma and breast cancer, an increased level of PKCe induces cell invasion and/or metastasis (24-26). Targeting $\mathrm{PKC} \varepsilon$ is considered to be a promising therapeutic method for cancer treatment. PKCe belongs to the PKC family of proteins, considered to be key signaling molecules in cellular functions $(25,26)$. Apoptosis, cell migration, proliferation, motility, chemoresistance and differentiation are examples of the cellular processes regulated by PKCs (27).
PKC $\varepsilon$ activates the Ras signaling cascade, which in turn leads to activation of cyclin D1 promoter, thus promoting cell survival and viability (28). PKCe has an anti-apoptotic function as activation of PKC $\varepsilon$ activates anti-apoptotic proteins B-cell lymphoma $(\mathrm{Bcl}) 2$ proteins and simultaneously suppressed the pro-apoptotic protein Bcl homology 3-interacting death agonist, thus inhibiting cell apoptosis $(29,30)$. It was also identified that expression of $\mathrm{PKC \varepsilon}$ is associated with resistance to chemotherapy in prostate and breast carcinoma $(25,31)$. In the present study, it was observed that tangeretin dose-dependently inhibited AGS cell viability. Furthermore, in the proteomic analysis of AGS cells treated with tangeretin, it was identified that PKCE expression was significantly downregulated. This result was further confirmed by western blot analysis. Furthermore, the PANTHER database also revealed that PKC $\varepsilon$ was the major protein involved in apoptosis and angiogenesis pathway (Table II). As PKCe has been identified to be an anti-apoptotic protein, the result that tangeretin treatment downregulated PKCe validates that tangeretin induces apoptosis of AGS cells through the PKC $\varepsilon$ signaling cascade.

PARP14 belongs to the PARP family of proteins. Previous studies have identified a protective function for PARP14 in lymphocytes against apoptosis and in hepatoma cells in vitro and in vivo $(32,33)$. PARP14 acts as a downstream protein of the c-Jun $\mathrm{N}$-terminal kinase (JNK) signaling pathway, thus further leading to cell survival in cancer cells. Barbarulo et al (34) concluded that JNK2 promotes myeloma cell survival via PARP14 which in turn inhibits JNK1, resulting in suppression of apoptosis in myeloma cells. In the present study, it was observed that tangeretin significantly inhibits PARP14 in tangeretin-treated AGS cells. The downregulation of PARP14 expression was further confirmed by western blot analysis. Thus, a significant decrease in PARP14 in tangeretin-treated AGS cells validated the inhibition of AGS cell viability and further induction of apoptosis.

MAPK4 belongs to the atypical MAPK family of proteins. Unlike the classical MAPK family proteins, the conserved T-X-Y motif is replaced by an S-E-G motif in MAPK4 (35). Mitogen-activated protein kinase-activated protein kinase 5 (MK5) is one of the best characterized downstream substrates of MAPK4. Overexpression of MAPK4 typically leads to activation of MK5 (36). MK5 is known to serve a function in tumor initiation and development. A function of the MAPK4/MK5 signaling pathway in insulin-like growth factor 2-binding protein 1-induced tumor cell migration has previously been identified (37). Suppression of MAPK4, being an upstream target of MK5, may inhibit tumor initiation and development. In the present study, MAPK4 expression was significantly suppressed in tangeretin-treated AGS cells, indicating its function as an antitumor marker.

Typically, cancer cells exhibit increased expression of PI4K. PI4K has been identified as a substrate for phosphoinositide 3-kinase and for producing secondary messengers (38). Knockdown of PI4K inhibits cell proliferation and induces apoptosis in breast cancer cells (39). It has been observed that PI4K acts as a mediator of resistance to cisplatin, thus inhibiting apoptosis in cancer cells (40). Thus, suppressing PI4K inhibits cell proliferation and induces apoptosis. Similarly, in the present study, it was observed that tangeretin inhibited PI4KA in AGS cancer cells, indicating the involvement of oncogenic protein degradation. 
The results of the present study demonstrated that tangeretin-treated human AGS cancer cells exhibit decreased viability and induction of cell death. Furthermore, proteomic changes in the cellular response towards tangeretin treatment in AGS cells have been identified. Differently expressed proteins identified functional genes that have been altered and significantly decreased. PKCE, MAPK4, PI4K and PARP14 proteins promote cell survival, tumor growth or development and suppression of apoptosis. The results of the present study demonstrated that tangeretin-induced cell death is regulated by the KPCE gene. Targeting KPCE may be a promising therapeutic marker in treating gastric cancer, thus tangeretin may be a useful therapeutic drug in gastric cancer treatment.

\section{Acknowledgements}

Not applicable.

\section{Funding}

The present study was supported by the National Research Foundation of Korea funded by Ministry of Science Industry and Technology (grant nos. 2012M3A9B8019303 and 2017R1A2B4003974).

\section{Availability of data and materials}

All the data generated or analyzed during this study are included in this published article.

\section{Authors' contributions}

SY, EHK, WSL and GSK conceived and designed the experiments. SY, SR and SMK performed the experiments and analyzed the data. VVGS, HJL, SEH, JDH, SJL and JAK contributed with reagents and data interpretation. SY, SR, JDH, SJL and JAK contributed in drafting and writing the manuscript. GSK, EHK and WSL drafted and critically reviewed the manuscript for important intellectual content. All authors read and approved the final manuscript.

\section{Ethics approval and consent to participate}

Not applicable.

\section{Patient consent for publication}

Not applicable.

\section{Competing interests}

The authors declare that they have no competing interests.

\section{References}

1. Nagini S: Carcinoma of the stomach: A review of epidemiology, pathogenesis, molecular genetics and chemoprevention. World J Gastrointest Oncol 4: 156-169, 2012

2. Luo C, Du Z, Wei X, Chen G and Fu Z: Bisdemethoxycurcumin attenuates gastric adenocarcinoma growth by inducing mitochondrial dysfunction. Oncol Lett 9: 270-274, 2015.
3. Ishii K, Tanaka S, Kagami K, Henmi K, Toyoda H, Kaise T and Hirano T: Effects of naturally occurring polymethyoxyflavonoids on cell growth, p-glycoprotein function, cell cycle, and apoptosis of daunorubicin-resistant $\mathrm{T}$ lymphoblastoid leukemia cells. Cancer Invest 28: 220-229, 2010.

4. Manthey JA and Guthrie N: Antiproliferative activities of citrus flavonoids against six human cancer cell lines. J Agric Food Chem 50: 5837-5843, 2002.

5. Martinez Conesa C, Vicente Ortega V, Yáñez Gascón MJ, Alcaraz Baños M, Canteras Jordana M, Benavente-García O and Castillo J: Treatment of metastatic melanoma B16F10 by the flavonoids tangeretin, rutin, and diosmin. J Agric Food Chem 53: 6791-6797, 2005.

6. Morley KL, Ferguson PJ and Koropatnick J: Tangeretin and nobiletin induce G1 cell cycle arrest but not apoptosis in human breast and colon cancer cells. Cancer Lett 251: 168-178, 2007.

7. Pan MH, Chen WJ, Lin-Shiau SY, Ho CT and Lin JK: Tangeretin induces cell-cycle G1 arrest through inhibiting cyclin-dependent kinases 2 and 4 activities as well as elevating Cdk inhibitors p21 and p27 in human colorectal carcinoma cells. Carcinogenesis 23: 1677-1684, 2002.

8. Dong Y, Cao A, Shi J, Yin P, Wang L, Ji G, Xie J and Wu D: Tangeretin, a citrus polymethoxyflavonoid, induces apoptosis of human gastric cancer AGS cells through extrinsic and intrinsic signaling pathways. Oncol Rep 31: 1788-1794, 2014.

9. Hong ML, Jiang N, Gopinath S and Chew FT: Proteomics technology and therapeutics. Clin Exp Pharmacol Physiol 33: 563-568, 2006

10. Grover A, Shandilya A, Bisaria VS and Sundar D: Probing the anticancer mechanism of prospective herbal drug Withaferin $\mathrm{A}$ on mammals: A case study on human and bovine proteasomes. BMC Genomics 11 (Suppl 4): S15, 2010.

11. Lu Z, Song Q, Yang J, Zhao X, Zhang X, Yang P and Kang J: Comparative proteomic analysis of anti-cancer mechanism by periplocin treatment in lung cancer cells. Cell Physiol Biochem 33: 859-868, 2014

12. Shevchenko A, Wilm M, Vorm O and Mann M: Mass spectrometric sequencing of proteins silver-stained polyacrylamide gels. Anal Chem 68: 850-858, 1996.

13. Szklarczyk D, Franceschini A, Kuhn M, Simonovic M, Roth A, Minguez P, Doerks T, Stark M, Muller J, Bork P, et al: The STRING database in 2011: Functional interaction networks of proteins, globally integrated and scored. Nucleic Acids Res 39: D561-D568, 2011.

14. Sangwan V and Park M: Receptor tyrosine kinases: Role in cancer progression. Curr Oncol 13: 191-193, 2006.

15. Hall A: The cytoskeleton and cancer. Cancer Metastasis Rev 28: 5-14, 2009.

16. Takai Y, Sasaki T and Matozaki T: Small GTP-binding proteins. Physiol Rev 81: 153-208, 2001.

17. Yu Y and Feng YM: The role of kinesin family proteins in tumorigenesis and progression: Potential biomarkers and molecular targets for cancer therapy. Cancer 116: 5150-5160, 2010.

18. Paul MK and Mukhopadhyay AK: Tyrosine kinase-Role and significance in cancer. Int J Med Sci 1: 101-115, 2004.

19. Berchtold MW and Villalobo A: The many faces of calmodulin in cell proliferation, programmed cell death, autophagy, and cancer. Biochim Biophys Acta 1843: 398-435, 2014.

20. Miao Z, Ali A, Hu L, Zhao F, Yin C, Chen C, Yang T and Qian A: Microtubule actin cross-linking factor 1, a novel potential target in cancer. Cancer Sci 108: 1953-1958, 2017.

21. Wang G, Tang J, Zhan W, Zhang R, Zhang M, Liao D, Wang X, Wu Y and Kang T: CBX8 suppresses tumor metastasis via repressing snail in esophageal squamous cell carcinoma. Theranostics 7: 3478-3488, 2017.

22. de Resende MF, Vieira S, Chinen LT, Chiappelli F, da Fonseca FP, Guimarães GC, Soares FA, Neves I, Pagotty S, Pellionisz PA, et al: Prognostication of prostate cancer based on TOP2A protein and gene assessment: TOP2A in prostate cancer. J Transl Med 11: 36, 2013.

23. Byun S, Lee KW, Jung SK, Lee EJ, Hwang MK, Lim SH, Bode AM, Lee HJ and Dong Z: Luteolin inhibits protein kinase C(epsilon) and c-Src activities and UVB-induced skin cancer. Cancer Res 70: 2415-2423, 2010.

24. Pal D, Outram SP and Basu A: Upregulation of PKC $\eta$ by $\mathrm{PKC} \varepsilon$ and PDK1 involves two distinct mechanisms and promotes breast cancer cell survival. Biochim Biophys Acta 1830: 4040-4045, 2013. 
25. Pan Q, Bao LW, Kleer CG, Sabel MS, Griffith KA, Teknos TN and Merajver SD: Protein kinase $\mathrm{C}$ epsilon is a predictive biomarker of aggressive breast cancer and a validated target for RNA interference anticancer therapy. Cancer Res 65: 8366-8371, 2005.

26. Selzer E, Okamoto I, Lucas T, Kodym R, Pehamberger H and Jansen B: Protein kinase $\mathrm{C}$ isoforms in normal and transformed cells of the melanocytic lineage. Melanoma Res 12: 201-209, 2002.

27. Gutcher I, Webb PR and Anderson NG: The isoform-specific regulation of apoptosis by protein kinase C. Cell Mol Life Sci 60: 1061-1070, 2003.

28. Kampfer S, Windegger M, Hochholdinger F, Schwaiger W, Pestell RG, Baier G, Grunicke HH and Uberall F: Protein kinase $\mathrm{C}$ isoforms involved in the transcriptional activation of cyclin D1 by transforming Ha-Ras. J Biol Chem 276: 42834-42842, 2001.

29. Ding L, Wang H, Lang W and Xiao L: Protein kinase C-epsilon promotes survival of lung cancer cells by suppressing apoptosis through dysregulation of the mitochondrial caspase pathway. J Biol Chem 277: 35305-35313, 2002.

30. McJilton MA, Van Sikes C, Wescott GG, Wu D, Foreman TL, Gregory CW, Weidner DA, Harris Ford O, Morgan Lasater A Mohler JL, et al: Protein kinase Cepsilon interacts with Bax and promotes survival of human prostate cancer cells. Oncogene 22: 7958-7968, 2003.

31. Wu D, Foreman TL, Gregory CW, McJilton MA, Wescott GG, Ford OH, Alvey RF, Mohler JL and Terrian DM: Protein kinase cepsilon has the potential to advance the recurrence of human prostate cancer. Cancer Res 62: 2423-2429, 2002.

32. Cho SH, Ahn AK, Bhargava $\mathrm{P}$, Lee $\mathrm{CH}$, Eischen $\mathrm{CM}$, McGuinness $\mathrm{O}$ and Boothby M: Glycolytic rate and lymphomagenesis depend on PARP14, an ADP ribosyltransferase of the B aggressive lymphoma (BAL) family. Proc Natl Acad Sci USA 108: 15972-15977, 2011.

33. Iansante V, Choy PM, Fung SW, Liu Y, Chai JG, Dyson J, Del Rio A, D'Santos C, Williams R, Chokshi S, et al: PARP14 promotes the Warburg effect in hepatocellular carcinoma by inhibiting JNK1-dependent PKM2 phosphorylation and activation. Nat Commun 6: 7882, 2015.
34. Barbarulo A, Iansante V, Chaidos A, Naresh K, Rahemtulla A, Franzoso G, Karadimitris A, Haskard DO, Papa S and Bubici C: Poly(ADP-ribose) polymerase family member 14 (PARP14) is a novel effector of the JNK2-dependent pro-survival signal in multiple myeloma. Oncogene 32: 4231-4242, 2013.

35. Aberg E, Perander M, Johansen B, Julien C, Meloche S, Keyse SM and Seternes OM: Regulation of MAPK-activated protein kinase 5 activity and subcellular localization by the atypical MAPK ERK4/MAPK4. J Biol Chem 281: 35499-35510, 2006.

36. Perander M, Al-Mahdi R, Jensen TC, Nunn JA, Kildalsen H, Johansen B, Gabrielsen M, Keyse SM and Seternes OM: Regulation of atypical MAP kinases ERK3 and ERK4 by the phosphatase DUSP2. Sci Rep 7: 43471, 2017.

37. Stöhr N, Köhn M, Lederer M, Glass M, Reinke C, Singer RH and Hüttelmaier S: IGF2BP1 promotes cell migration by regulating MK5 and PTEN signaling. Genes Dev 26: 176-189, 2012.

38. Balla A and Balla T: Phosphatidylinositol 4-kinases: Old enzymes with emerging functions. Trends Cell Biol 16: 351-361, 2006.

39. Chu KM, Minogue S, Hsuan JJ and Waugh MG: Differential effects of the phosphatidylinositol 4-kinases, PI4KII $\alpha$ and PI4KIII $\beta$, on Akt activation and apoptosis. Cell Death Dis 1: e106, 2010

40. Waugh MG: Phosphatidylinositol 4-kinases, phosphatidylinositol 4-phosphate and cancer. Cancer Lett 325: 125-131, 2012.

This work is licensed under a Creative Commons Attribution-NonCommercial-NoDerivatives 4.0 International (CC BY-NC-ND 4.0) License. 